\title{
PRINCIPIOS FORMALES*
}

Robert Alexy

Universidad de Kiel

RESUMEN. En este trabajo se defiende la teoría principialista de los derechos fundamentales de las críticas que la acusan de ser incapaz de determinar de forma adecuada la relación entre los derechos fundamentales y el control constitucional, con la democracia. Para llegar a una adecuada teoría de la discrecionalidad legislativa, se necesita analizar el papel de los principios formales en la ponderación. Para ello se defiende un «modelo epistémico» de ponderación entre principios formales y sustanciales que se situaría entre el llamado «modelo sustancial-formal puro» y el «modelo combinado», y que se fundamentaría en la idea de ponderación de segundo orden, incorporando la fiabilidad epistémica en la fórmula del peso.

Palabras clave: Principios formales, proporcionalidad, discrecionalidad legislativa, fórmula del peso, ponderación.

\section{Formal Principles}

ABSTRACT. This paper argues in favor of the principle theory of fundamental rights. It is an elaborated reaction against those objections considering the theory not being able to determine the appropriate relation between fundamental rights, constitutional control, and democracy. According to the author, in order to construct an adequate theory of legislative discretion, we must analyze the role that formal principles play in balancing. As a methodological instrument, the author defends an «epistemic model» for the balancing of formal and substantial principles. This model should be considered between the so called «pure substantial-formal model» and «a combined model». The base of this model is to be found in the idea of second order balancing, incorporating the variable of epistemic fidelity in the weight formula.

Keywords: Formal principles, proportionality, legislative discretion, weight formula, balancing.

* Fecha de recepción: 23 de septiembre de 2014. Fecha de aceptación: 21 de octubre de 2014.

Me gustaría agradecer a Stanley L. PAULSON por su ayuda y recomendaciones para la redacción de la versión en inglés. 


\section{EL PROBLEMA}

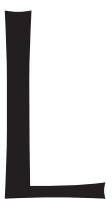

a teoría de los derechos fundamentales como principios ha recibido numerosas objeciones ${ }^{1}$. En el ámbito del constitucionalismo democrático la objeción institucional cobra especial importancia. Esta objeción señala que la tesis de la optimización — sin la cual la teoría de los principios no sería la teoría de los principios- conduce a una eficacia expansiva de los derechos fundamentales, llevando a su vez a una sobre-constitucionalización del sistema jurídico. Según Ernst-Wolfgang BÖCKENFÖRDE, la consecuencia institucional es un «desplazamiento del estado legislativo parlamentario al estado de adjudicación constitucional» ${ }^{2}$. Brevemente, el reproche a la teoría de los principios se centra en señalar que es incapaz de determinar de forma adecuada, por un lado, la relación entre los derechos fundamentales y el control constitucional, y, por otro lado, de éstos con la democracia.

La teoría de los principios ha intentado desde sus inicios contestar a esta objeción con una teoría de la discrecionalidad. En esta teoría, el concepto de principio formal juega un rol fundamental. Este concepto, sin embargo, es muy controvertido todavía. La controversia sobre los principios formales no es solamente parte importante del debate entre los opositores y los defensores de la teoría de los principios, sino que también ha llegado a existir una agitada disputa acerca de los principios formales incluso dentro del campo de la teoría de los principios.

Martin BOROWSKI recientemente ha caracterizado los principios formales como un elemento de la teoría de los principios que «curiosamente ha permanecido como un enigma» ${ }^{3}$, y se refiere a ellos con la metáfora de «el último gran parche blanco en el mapa de la teoría de los principios» ${ }^{4}$. Intentaré defender la tesis según la cual es posible que un análisis de los principios formales implique una adecuada teoría de la discrecionalidad legislativa. El fundamento de este modelo es la fórmula del peso. Debo empezar con una presentación de algunos elementos básicos de la teoría de los principios.

${ }^{1}$ Sobre una clasificación de estas objeciones en siete grupos vid. R. ALEXY, «The Construction of Constitutional Rights», en Law E Ethics of Human Rights 4, 2010, 24-5; Sobre la teoría de principios en general vid., id., «On the Structure of Legal Principles», en Ratio Juris 13, 2000, 294-304; id., «Constitutional Rights, Balancing, and Rationality», en Ratio Juris 16, 2003, 131-140; J. RIVERS, «Proportionality, Discretion and the Second Law of Balancing», en G. Pavlakos (ed.), Law, Rights and Discourse. The Legal Philosophy of Robert Alexy, Oxford, Hart Publishing, 2007, 167-188; M. JESTAEDT, «The Doctrine of Balancing - its Strengths and Weaknesses», en M. KLATT (ed.), Institutionalized Reason. The Jurisprudence of Robert Alexy, Oxford, Oxford University Press, 2012, 152-172.

2 E.-W. BÖCKENFÖRDE, Staat, Verfassung, Demokratie, Frankfurt-on-Main, Suhrkamp, 1991, 190.

3 M. Borowski, «Formelle Prinzipien und Gewichtsformel», en M. KlaTT (ed.), Prinzipientheorie und Theorie der Abwägung, Tübingen, Mohr Siebeck, 2013, 151.

4 Ibid. 


\section{ALGUNOS ELEMENTOS BÁSICOS DE LA TEORÍA DE LOS PRINCIPIOS}

\subsection{Reglas y Principios}

La base de la teoría de principios es la distinción teórica entre reglas y principios ${ }^{5}$. Las reglas son normas que requieren estar determinadas. Son mandatos definitivos. Su forma de aplicación es la subsunción. Por el contrario, los principios son mandatos de optimización. De por sí, estos exigen «que algo sea realizado en la mayor medida posible dentro de las condiciones fácticas y jurídicas existentes» ${ }^{6}$. Además de las reglas, las posibilidades jurídicas están determinadas esencialmente por los principios en oposición. Por tal razón, los principios, considerados cada uno de ellos por separado, siempre se componen simplemente de mandatos prima facie. La determinación del grado apropiado de satisfacción de un principio en relación con aquello exigido por otros principios se logra a través de la ponderación. Así, la ponderación es la forma específica de aplicación de los principios.

\section{Proporcionalidad}

La naturaleza de los principios como mandatos de optimización, conduce enseguida a una conexión necesaria entre principios y análisis de proporcionalidad. El principio de proporcionalidad, el cual en las últimas décadas ha recibido el mayor reconocimiento internacional de todos los tiempos tanto en el ámbito práctico como teórico del control constitucional ${ }^{7}$, consiste en tres sub-principios: el principio de idoneidad, el de necesidad y el de proporcionalidad en sentido estricto. Los tres sub-principios expresan la idea de optimización. Por esta razón, la naturaleza de los principios implica el principio de proporcionalidad y viceversa.

Los principios de idoneidad y necesidad se refieren a la optimización en relación con las condiciones fácticas. Esta optimización relativa a las posibilidades fácticas existentes consiste en evitar costos evitables ${ }^{8}$. Costos que, sin embargo, son inevitables cuando hay principios que colisionan. Entonces la ponderación se hace necesaria. La ponderación es objeto del tercer sub-principio del principio de proporcionalidad, específicamente del principio de proporcionalidad en sentido estricto. Este principio expresa lo que significa la optimización en relación con las condiciones jurídicas. Lo cual es idéntico a la regla que podría llamarse «la ley de la ponderación» ${ }^{9}$. Esta ley establece:

Cuanto mayor es el grado de insatisfacción o detrimento de un principio, tanto mayor debe ser la importancia de satisfacción del otro.

\footnotetext{
${ }^{5}$ R. AleXY, A Theory of Constitutional Rights (first publ. 1985), trad. J. RivERS, Oxford, Oxford University Press, 2002, 47-48.

${ }^{6}$ Ibid. 47.

7 Vid., por ejemplo: D. M. BEATTY, The Ultimate Rule of Law, Oxford, Oxford University Press, 2004; A. Stone SweEt y J. MATHEWS, «Proportionality Balancing and Global Constitutionalism», Columbia Journal of Transnational Law, 47, 2008, 72-164; A. BARAK, Proportionality, Constitutional Rights and their Limitation, Cambridge, Cambridge University Press, 2012.

8 R. Alexy, «Constitutional Rights and Proportionality», Chinese Yearbook of Constitutional Law, 2010, 222-224.

9 Alexy, A Theory of Constitutional Rights, n. 5 supra, 102.
} 
Robert Alexy

\subsection{Fórmula del peso}

Casi en cualquier lugar en el ámbito de la adjudicación constitucional, se encuentra la ley de la ponderación en varias y diferentes formulaciones. Esta expresa la esencia de la ponderación y es por ello de gran importancia práctica. El análisis de los principios formales y la discrecionalidad legislativa, requiere, sin embargo, una descripción más precisa y completa de la estructura de la ponderación. Con el fin de lograr tal descripción, la ley de la ponderación tiene que ser más elaborada. El resultado de esta mayor elaboración es la fórmula del peso ${ }^{10}$. Esta fórmula opera de la siguiente forma:

$$
W_{i, j}=\frac{I_{i} \cdot W_{i} \cdot R_{i}}{I_{j} \cdot W_{j} \cdot R_{j}}
$$

$W_{i, j}$ representa el peso concreto del principio $P_{i}$ en relación con el principio en colisión $P_{j}$. La fórmula del peso define este peso concreto como el cociente de los tres factores fijados, por así decir, en cada lado de la ponderación. $I_{i}$ e $I_{j}$ son de especial importancia. $I_{i}$ fija la intensidad de la interferencia con $P_{i} . I_{j}$, representa la importancia de satisfacer el principio en colisión $P_{j} . I_{j}$, también, puede entenderse como intensidad de interferencia, esto es, como la intensidad de la interferencia con $P_{j}$ a través de la no-interferencia con $P_{i}$. $W_{i}$ y $W_{j}$ establecen los pesos abstractos de los principios en colisión $P_{i}$ y $P_{j}$. Cuando los pesos abstractos son iguales, lo que ocurre con frecuencia en el caso de colisión o conflicto de derechos fundamentales, éstos se neutralizan entre sí.

$I_{i}$ e $I_{j}$, y también $W_{i}$ y $W_{j}$, corresponden a la dimensión material de la ponderación. $R_{i}$ y $R_{j}$ tienen un carácter completamente diferente. Se refieren a la fiabilidad de los supuestos empíricos y normativos, con respecto a, primero y ante todo, la cuestión de cuán intensa es la interferencia con $P_{i}$, y cuán intensa sería la interferencia con $P_{j}$, si la interferencia con $P_{i}$ fuera omitida. Además de esto, la fiabilidad de los supuestos empíricos y normativos también puede identificarse con la clasificación de los pesos abstractos, esto es, $W_{i}$ y $W_{j}$. El punto decisivo es que la fiabilidad es un factor que no se refiere a las cosas, en nuestro caso la intensidad de interferencia y los pesos abstractos, es decir, no es un factor óntico. Es, más bien, un factor referido al conocimiento de las cosas. Esto quiere decir que es un factor epistémico. Esta distinción entre factores ónticos y epistémicos se tornará de gran importancia para la teoría de los principios formales y la discrecionalidad legislativa.

Desde $2003^{11}$, la fiabilidad ha sido representada en ambos lados de la fórmula del peso sólo por una variable: $R_{i}$ y $R_{j}$, y en algunas publicaciones he definido $R_{i}$ y $R_{j}$ como referidas sólo a «supuestos empíricos» ${ }^{12}$. Esto sin embargo no encaja bien con el hecho de que en el epílogo de «La teoría de los derechos fundamentales», esto es, en 2002, ya había enfatizado la importancia sistemática de la distinción entre discrecionalidad empírico-epistémica y discrecionalidad normativo-epistémica ${ }^{13}$. Esta diferencia tam-

10 R. Alexy, «The Weight Formula», en J. Stelmach, B. BroŻEK y W. ZaŁusKi (eds.), Frontiers of the Economic Analysis of Law, Krakow, Jagiellonian University Press, 2007, 25.

11 R. AleXY, «On Balancing and Subsumtion. A Structural Comparison», en Ratio Juris, 16, $2003,446$. Para una anterior notación, vid. ALEXY, A Theory of Constitutional Rights, n. 5 supra, 419.

12 AleXY, A Theory of Constitutional Rights, n. 5 supra, 419; id., «On Balancing and Subsumption», n. 11 supra, 446; id., «The Weight Formula», n. 10 supra, 25.

13 Alexy, A Theory of Constitutional Rights, n. 5 supra, 414-425. 
poco se articula muy bien con la formulación de la segunda ley de la ponderación, esto es, la formulación de la ley epistémica de la ponderación, la cual establece:

Cuanto más intensa sea la interferencia en un derecho constitucional, tanto mayor debe ser la certeza de sus premisas subyacentes ${ }^{14}$.

Esta ley se refiere no sólo a las premisas empíricas sino, en una forma muy general, a las «premisas subyacentes». Ahora el punto decisivo es que este concepto comprende tanto premisas normativas como premisas empíricas. $R_{i}$ y $R_{j}$ deben por tanto entenderse como referidas a premisas normativas así como a premisas empíricas. Esto puede expresarse en la siguiente ecuación:

$$
R_{i}=R_{i}^{e} \cdot R_{i}^{n}
$$

Esta ecuación podría llamarse «ecuación de fiabilidad».

He caracterizado la fórmula del peso antes presentada, como la «Fórmula del Peso Completa» ${ }^{15}$, y puede mantenerse bajo ese nombre. En un gran número de casos, la fiabilidad empírica considerada de forma aislada es un problema. Entonces, no hay necesidad práctica de aplicar explícitamente su ecuación. Sin embargo, tan pronto como estén en cuestión tanto la fiabilidad empírica como la normativa, $R_{i}$ and $R_{j}$ deben ser sustituidas por los productos respectivos en concordancia con la ecuación de fiabilidad. De esta forma, una versión refinada de la fórmula del peso entra en escena:

$$
W_{i, j}=\frac{I_{i} \cdot W_{i} \cdot R_{i}^{e} \cdot R_{i}^{n}}{I_{j} \cdot W_{j} \cdot R_{j}^{e} \cdot R_{j}^{n}}
$$

Esta versión podría designarse como la «Fórmula del peso completa y refinada». Un considerable número de autores han sugerido que una cuarta variable debe introducirse en la fórmula del peso ${ }^{16}$. En la medida en la cual estas propuestas sean compatibles con la ecuación de fiabilidad y con el refinamiento de la fórmula del peso lograda, estoy de acuerdo con ellos.

Una fórmula como la del peso, que expresa el cociente de dos productos, es sensata sólo si todos los factores pueden ser representados por números. Este es el problema de la gradación. En otra parte ${ }^{17}$ he propuesto una escala discreta, esto es, una escala tríadica discontinua, en la cual las secuencias geométricas son implementadas. Esta escala asigna los rangos «leve», «moderado» y «serio» a la intensidad de la interferencia y a los pesos abstractos. Estos valores son expresados por las cifras $2^{0}, 2^{1}$, y $2^{2}$, es decir, por 1,2 , y 4 . Donde el factor epistémico está relacionado con $R_{i}$ y $R_{j}$, o, en la versión refinada de la fórmula del peso, $R_{i}^{e}$ y $R_{i}^{n}$ así como $R_{j}^{e}$ y $R_{j}^{n}$, se puede trabajar con los escenarios de «fiabilidad» o «certeza» (f), plausibilidad (p), y no evidentemente falso (e),

14 Ibid., 418.

15 ALEXY, «The Weight Formula», n. 10 supra, 25.

16 Vid., por ejemplo, J. BADENHOP, Normtheoretische Grundlagen der Europäischen Menschenrechtskonvention, Baden-Baden, Nomos, 2010, 366-367; M. KLATT y M. MEISTER, The Constitutional Structure of Proportionality, Oxford, Oxford University Press, 2012, 132; M. KLATT y J. SCHMIDT, «Epistemic discretion enconstitutional law», en International Journal of Constitutional Law, 10, 2012, 91; J. A. PORTOCARRERO QUISPE, Der autoritative Charakter der Grundrechtsabwägung, en prensa, part 1, section 2, IV 6.3.

17 AleXY, A Theory of Constitutional Rights, n. 4 supra, 409-410, 419; id., «The Weight Formula», n. supra, 20-26. 
para los cuales las cifras $2^{0}, 2^{-1}$, y $2^{-2}$, es decir, $1, \frac{1}{2}$, y $\frac{1}{4}$, están por asignarse ${ }^{18}$. Si los valores epistémicos ascienden a 1 , los valores materiales permanecen intactos. Si los valores epistémicos son menores que 1, el impacto de los valores materiales o sustanciales se reduce en consecuencia. El menor valor epistémico se da cuando la fiabilidad tanto de las premisas empíricas como normativas es evaluado no tan alto como «no evidentemente falso». En este caso, el lado del valor epistémico recibe el valor de $\frac{1}{16}$. Tal cualidad extremadamente nociva destruye el poder incluso de las más fuertes razones sustanciales para interferir con derechos fundamentales casi por completo. Esto demuestra que la ecuación de fiabilidad tiene una gran plausibilidad intuitiva.

Por medio de estas tríadas, la mayoría de las decisiones de las cortes constitucionales pueden comprenderse. Donde no sean suficientes, esto es, donde se tiene que introducir una gradación aún más atenuada, estas pueden extenderse a escalas triádicas dobles ${ }^{19}$. Mucho más puede decirse de la fórmula del peso. Para una discusión de los principios formales, sin embargo, lo dicho hasta aquí debe ser suficiente.

\section{EL CONCEPTO DE PRINCIPIO FORMAL}

Los principios formales son principios. Los principios son mandatos de optimización. Así, la definición de mandatos de optimización como «normas que requieren que algo se realice en la mayor medida posible de acuerdo a las condiciones fácticas y jurídicas reales existentes» ${ }^{20}$ aplica a los principios formales de la misma forma que a los principios sustanciales. La diferencia entre estas dos clases de principios está restringida por aquello a lo que la palabra «algo» se refiere en la definición, es decir, al objeto de optimización. La differentia specifica de los principios sustanciales o materiales es que sus objetos de optimización son ciertos contenidos, como, por ejemplo, la vida, libertad de expresión, el mínimo vital, y la protección del medio ambiente. Por el contrario, los objetos de optimización de los principios formales son decisiones legales sin tener en cuenta su contenido. Los principios formales requieren que la autoridad de las normas debidamente expedidas y socialmente eficaces sea optimizada. La expedición autoritativa y la eficacia social son elementos definitorios del positivismo jurídico $^{21}$. Esto implica que los principios formales se refieren a la dimensión real o fáctica del derecho.

Este aspecto real o fáctico puede, sin embargo, conectarse con elementos ideales. El principio democrático es un ejemplo. La democracia es un intento de institucionalizar los ideales del discurso qua deliberación pública en lo que sea posible ${ }^{22}$. Sin embargo, éste es un principio formal. Se refiere, en lo que respecta a la democracia representativa, no a la democracia directa, sino a las decisiones tomadas por el parlamento según la regla de la mayoría. La democracia, sin embargo, no es simplemente

\footnotetext{
18 Id., «The Weight Formula», n. supra, 25.

19 Ibid., 22-23.

20 ALEXY, A Theory of Constitutional Rights, n. 5 supra, 47.

21 R. AleXY, The Argument from Injustice. A Reply to Legal Positivism (primera publicación 1992), trad. B. Litschewski Paulson y S. L. Paulson, Oxford, Clarendon Press, 2002, 3-4, 13-19.

22 R. Alexy, «The Dual Nature of Law», en Ratio Juris, 23, 2010, 177-178.
} 
un procedimiento de decisión entre otros. Éste es el procedimiento más racional y legítimo para la expedición de normas. Por esta razón, el principio democrático otorga no sólo un especial peso a las decisiones del parlamento. Dicho principio exige, por encima de todo, «que el legislador democráticamente legitimado debe tomar tantas decisiones importantes para la sociedad como sea posible» ${ }^{23}$. Con esta exigencia, el principio de la democracia se refiere no sólo a la autoridad de las decisiones tomadas desde el ámbito de competencia del parlamento. También demanda que dicho ámbito sea tan amplio como sea posible. Exigencia de especial importancia para la relación entre derechos fundamentales y democracia.

\section{PRINCIPIOS Y PONDERACIÓN EN GENERAL}

T. R. S. AlLEN ha sostenido que «un principio democrático puramente formal [...] carece de peso independiente» ${ }^{24}$. De aquí surge la pregunta de si los principios formales pueden participar en la ponderación como lo describe la fórmula del peso. Aceptaré, en primer lugar, esta pregunta con respecto a los principios formales y sustanciales en general. La pregunta sobre ponderar democracia y derechos fundamentales la tendré en cuenta más adelante. Ahora la pregunta es: ¿Puede un principio formal ponderarse contra un principio sustancial, y viceversa?

Es fácil encontrar casos en los cuales un principio formal puede, e incluso debe, ponderarse contra un principio material. Después del Nacional Socialismo que fue derrotado en 1945, así como después del colapso de la República Democrática Alemana en 1989, los tribunales en Alemania se enfrentaron en cierto número de casos con el problema de los estatutos injustos. Éstos aplicaron la fórmula de RADBRUCH, la cual en su forma más corta, opera como sigue:

La extrema injusticia no es derecho ${ }^{25}$.

Esta fórmula es el resultado de ponderar el principio material de justicia contra el principio formal de seguridad jurídica. ${ }^{26} \mathrm{La}$ ponderación descansa en dos leyes. La primera ha sido de hecho introducida. Ésta es la ley de la ponderación, la cual encuentra su forma más elaborada en la fórmula del peso. La segunda es la ley de los principios en colisión ${ }^{27}$, la cual señala que según el peso concreto del principio en colisión $P_{i}$, establecido por la fórmula del peso $\left(W_{i, j}\right)$, una relación concreta de preferencia tiene que establecerse con respecto a las condiciones del caso. La fórmula de RADBRUCH descansa en la distinción entre dos condiciones de precedencia. La primera, $C_{1}$, es injusticia bajo el umbral de la extrema injusticia. La segunda, $C_{2}$, es extrema injusticia. Bajo la primera condición, $C_{1}$, el principio formal de seguridad jurídica tiene precedencia sobre el principio material de justicia. Bajo la segunda condición $C_{2}$, el principio material de justicia tiene precedencia sobre el principio formal de seguridad jurídica. Según la ley

23 AleXy, A Theory of Constitutional Rights, n. 5 supra, 417.

24 T. R. S. AlLEN, «Constitutional Rights and the Rule of Law», en KLATT (ed.), Institutionalized Reason, n. 1 supra, 135-136.

${ }_{25}$ R. AleXY, «A Defence of Radbruch's Formula», en M. D. A. FrEeman (ed.), Lloyd's Introduction to Jurisprudence, 8th ed., London, Sweet \& Maxwell and Thomson Reuters, 2008, 428.

26 R. ALEXY, «The Dual Nature of Law», n. 22 supra, 177.

27 Alexy, A Theory of Constitutional Rights, n. 5 supra, 54. 
de los principios en colisión, la consecuencia de la precedencia del principio de justicia sobre el principio de seguridad jurídica bajo la condición de extrema injusticia es que bajo esta condición la consecuencia requerida por el principio dominante de justicia es aplicable, y es exactamente lo que la fórmula de RADBRUCH enuncia.

Es de la máxima importancia que todo esto se conecte intrínsecamente con la fórmula del peso. El principio formal de seguridad jurídica tiene precedencia sobre el principio material de justicia si, y sólo si, tiene el peso concreto mayor. Ahora, en la condición de injusticia por debajo del umbral de la extrema injusticia, la interferencia con el principio de justicia no tiene la mayor intensidad. Yo clasificaría esto, en la base de la escala tríadica, en el mejor de los casos en el medio, y en casos de menor injusticia incluso como leve. El valor de la seguridad jurídica por debajo del umbral de la extrema injusticia es, sin embrago, alto. Si todas y cada una de las injusticias condujeran a la invalidez jurídica, la función del sistema jurídico de coordinar el comportamiento y asegurar las ventajas de la coordinación y cooperación social se extinguiría. Según la fórmula del peso, un principio con un mayor peso justifica una interferencia a un principio con peso medio o leve. Si, sin embargo, la injusticia es extrema, la interferencia con el principio de justicia sería grave. Al contrario se tiene que asumir sólo una interferencia media o leve al principio de la seguridad jurídica. La extrema injusticia ocurre sólo en extremas situaciones de los sistemas jurídicos, y esto de ninguna manera concierne a todas las normas jurídicas, sino sólo algunas específicas de todo el sistema jurídico ${ }^{28}$. Todo esto quiere decir que la ponderación de principios formales y sustanciales no es solamente posible, sino que, de hecho, es también necesaria en el derecho.

Algo similar sucede cuando un sistema jurídico permite decisiones contra legem, al menos en algunas áreas del derecho, como lo hace el sistema jurídico alemán. Una norma no es automáticamente desplazada cuando un principio sustancial contrario tiene mayor peso que su propio principio sustancial subyacente en los hechos del caso. Una decisión contra legem, por ejemplo la incorporación de una excepción a una norma en un caso particular a decidir, requiere que el principio sustancial contrario tenga un peso mayor que el principio sustancial subyacente a la norma sumado a los principios formales de seguridad jurídica, el principio democrático y el de separación de poderes, todo lo cual requiere que lo que ha sido autoritativamente expedido debe respetar$\mathrm{se}^{29}$. Esto demuestra que los principios formales no sólo pueden ponderarse en contra de sus propios principios sustanciales en colisión, como con la noción de la fórmula de RADBRUCH, sino que también pueden ponderarse en combinación con principios materiales contra principios materiales en colisión. El primer modelo se podría llamar «modelo sustancial-formal puro» de ponderación de principios y el segundo «modelo mixto sustancial-formal», o, más corto, «modelo combinado».

\section{LA VÍA EQUIVOCADA}

Se podría pensar que estos dos modelos también se aplican a la relación entre derechos fundamentales y el principio formal de la democracia. De hecho, en la Teoría

${ }^{28}$ ALEXY, The Argument from Injustice, n. 21 supra, 66-68.

29 AleXY, A Theory of Constitutional Rights, n. 5 supra, 58. 
de los derechos fundamentales por primera vez publicada en 1985, respaldé el modelo combinado:

Un principio formal o procedimental es, por ejemplo, el principio que dice que el legislador democrático tomará decisiones que son significativas para la sociedad como un todo. Este principio procedimental, junto con un principio material que sirve como algún interés público secundario, puede ser ponderado contra un principio constitucional para garantizar un derecho individual ${ }^{30}$.

Más recientemente, BOROWSKI ha argumentado a favor del modelo combinado:

La consideración del principio formal tiene el efecto de dar peso a uno de los dos principios materiales ${ }^{31}$.

Por esta razón:

Además de los principios materiales "i" e "j" un principio "f" debe insertarse en la fórmula del peso ${ }^{32}$.

Tanto el «modelo sustancial-formal puro» como «el modelo combinado» conducen, sin embargo, a serios problemas en lo que respecta a los derechos fundamentales. Esto se ve con especial claridad en el caso del «modelo sustancial-formal puro». Si el principio democrático en sí mismo fuera capaz de justificar una interferencia con un derecho fundamental, sería posible decir: «La interferencia no está justificada por algún principio material, es decir, no está justificada por alguna razón material, no obstante es permisible, para que el legislador democráticamente legitimado pueda tomar más decisiones cuando dicha interferencia está permitida que cuando no». Una interferencia con un derecho fundamental que no está justificada por una razón material, no es solamente desproporcionada, es también arbitraria. Reconocer una competencia al legislador para interferir con los derechos fundamentales simplemente por ser democráticamente legitimado debilitaría la prioridad de la constitución sobre la legislación parlamentaria ordinaria.

Las cosas son más complejas en el caso del «modelo mixto sustancial-formal», o «modelo combinado». Sin embargo, este modelo tiene, básicamente, los mismos defectos que el «modelo sustancial-formal puro». Si se insertaran principios formales en la fórmula del peso junto a los principios materiales combinaciones como la siguiente serían posibles. De un lado, la interferencia con un derecho fundamental es grave $(g)$, esto es, $I_{i}$ tiene valor de 4 . Del otro lado, la importancia de satisfacer el principio en colisión es moderada $(m)$, es decir, $I_{j}$, tiene valor de 2 . Éste es un caso claro de desproporcionalidad, y con ello, de inconstitucionalidad. Ahora es posible atribuir al principio formal de competencia decisoria del legislador, estando del lado del principio material en colisión, un mayor valor, esto es, $P_{f}$ recibe el valor de 4 . De este modo se transformaría una decisión inconstitucional en constitucional. Si esto fuera posible, interferencias desproporcionadas con los derechos fundamentales serían permisibles, con fundamento en que el legislador democráticamente legitimado puede tomar más decisiones cuando esto está permitido que cuando no lo está. Como en el caso del

30 Ibid., 82 (énfasis añadido).

31 M. BOROWSKI, «The Structure of Formal Principles - Robert Alexy's Law of Combination», en Archives for Philosophy of Law and Social Philosophy, supplement 119, 2010, 34.

32 BorowsKI, «Formelle Prinzipien und Gewichtsformel», n. 3 supra, 184. 
«modelo sustancial-formal puro», se debilitaría la prioridad de la constitución sobre la legislación parlamentaria ordinaria.

$¿$ Esto significa que el legislador democrático nunca tiene discrecionalidad respecto de los derechos fundamentales? La respuesta es: no. La comprobación de esta respuesta es posible con base en el análisis del concepto de discrecionalidad.

\section{DOS CLASES DE DISCRECIONALIDAD}

Existen dos clases de discrecionalidad: sustancial o estructural y epistémica. La discrecionalidad sustancial del legislador se compone de todo lo que las normas constitucionales no pueden de forma definitiva ni prohibir ni ordenar. Lo que no está prohibido ni es ordenado está permitido ${ }^{33}$. Así el ámbito de discrecionalidad sustancial es idéntico a lo que la constitución ha dejado definitivamente a liberalidad del legislador. La cuestión de la discrecionalidad epistémica surge siempre que el conocimiento sobre lo que está definitivamente prohibido, ordenado, o permitido por las normas constitucionales es incierto. En tales casos el legislador tiene discrecionalidad epistémica hasta el grado de determinar qué es lo que está definitivamente prohibido, ordenado, o permitido de acuerdo a su competencia. La discrecionalidad sustancial, la cual existe en tres formas ${ }^{34}$, es mucho menos problemática que la discrecionalidad epistémica. Que el legislador sea libre donde no existen obligaciones no requiere justificación. Por tal razón, los principios formales no juegan un papel con respecto a la discrecionalidad sustancial. Que el poder legislativo tenga competencia para decidir si es libre o no en casos en los cuales tal libertad es incierta es, sin embargo, mucho menos obvio. Aquí es donde los principios formales juegan un papel decisivo. En lo que sigue se considerará sólo la discrecionalidad epistémica.

\section{SEGUNDO ORDEN DE OPTIMIZACIÓN EPISTÉMICA}

La discrecionalidad epistémica tiene que clasificarse más a fondo en dos clases: empírica y normativo-epistémica. La discrecionalidad empírica genera muchos menos problemas que la discrecionalidad normativo-epistémica. Por tanto, la discrecionalidad empírica será el centro de nuestras consideraciones ${ }^{35}$.

La incertidumbre empírica puede ser un problema en los tres escenarios del análisis de proporcionalidad. La decisión sobre el cannabis del Tribunal Constitucional Alemán nos ofrece un buen ejemplo. La cuestión central de esta decisión está orientada a establecer qué peligros están asociados con el uso de los productos derivados del cannabis, y a través de qué medidas pueden combatirse. El legislador ha justificado su prohibición sobre los productos de cannabis, con la expedición de sanciones penales, bajo el supuesto de que un mayor riesgo para la salud y la vida social sólo puede

33 AlEXy, A Theory of Constitutional Rights, n. 5 supra, 130-131.

34 Ibid., 395-414.

35 Sobre discrecionalidad normativo-epistémica, véase ALEXY, A Theory of Constitutional Rights, n. 5 supra, 415-416, 420-422. 
combatirse adecuadamente mediante leyes penales ${ }^{36}$. La contraparte argumentó que el mayor riesgo asumido por el legislador no existe. El riesgo es en el mejor de los casos mínimo, y las medidas tomadas por el legislador no son ni idóneas ni necesarias. Ahora se podría considerar la visión según la cual el Tribunal debe permitir la interferencia en el derecho fundamental sólo si se ha establecido la veracidad de aquellos supuestos empíricos de los que dependen la idoneidad, la necesidad, y el alcance del riesgo. Pero el Tribunal procede de forma distinta. No establece la veracidad o falsedad de las premisas empíricas del legislador, sino su incertidumbre: «No se dispone de una base de conocimientos científicamente fundados, que necesariamente apunte hacia la corrección de una u otra alternativa» ${ }^{37}$. No obstante, el Tribunal permite la interferencia con el derecho fundamental. Éste es el resultado de reconocer al legislador discrecionalidad con respecto al conocimiento de los hechos relevantes, en otras palabras, una discrecionalidad empírico-epistémica, ubicando además los supuestos empíricos del legislador subyacentes a la prohibición del cannabis dentro de esta discrecionalidad.

El juicio del cannabis expone de forma clara el principal problema con la discrecionalidad epistémica. Si el legislador está autorizado para basar su intervención sobre un derecho fundamental en premisas inseguras, entonces es posible que la protección dada por los derechos fundamentales sea denegada con base en estimaciones falsas, a pesar de que en realidad el derecho sea vulnerado.

La solución la ofrece un tercer modelo en relación con el papel que juegan los principios formales en la ponderación, un modelo que se ubica en el punto intermedio entre «modelo sustancial-formal puro» y el «modelo combinado o mixto sustancialformal». Este tercer modelo puede llamarse «modelo epistémico». El fundamento del modelo epistémico es la idea de ponderación de segundo orden. La ponderación según los lineamientos de la fórmula del peso es de primer orden. La ponderación de segundo orden concierne a la justificación de incorporar la fiabilidad epistémica, que es, de $R_{i}$ and $R_{i}$, en la fórmula del peso ${ }^{38}$. Los derechos fundamentales como principios requieren la máxima realización posible considerando las condiciones fácticas y jurídicas dadas. La realización de los derechos fundamentales se incrementa cuando éstos sólo pueden limitarse sobre el terreno de premisas cuya verdad está acreditada. Si se admitieran premisas por debajo del nivel de certeza, sin embargo, la realización de los derechos fundamentales aumentaría si se escogieran las premisas más favorables al derecho fundamental. La discrecionalidad del legislador se excluiría también en este caso. En este sentido, los derechos fundamentales requieren no sólo una optimización sustancial, sino también una optimización epistémica ${ }^{39}$. El punto decisivo en la ponderación de segundo orden es que los derechos fundamentales como mandatos epistémicos de optimización colisionan con el principio formal del legislador democrático. Si esta colisión pudiera resolverse estableciendo una precedencia absoluta del principio sustancial de los derechos fundamentales sobre el principio formal del legislador democrático, las consecuencias serían inaceptables en amplias áreas del derecho. En contextos razonablemente complejos, como el derecho comercial, la seguridad y el

\footnotetext{
36 BVerfGE 90, 145, 174.

37 BVerfGE 90, 145, 182-183.

38 En la fórmula refinada del peso $R_{i}$ es sustituido por $R_{i e} \cdot R_{i n}$, y $R_{j}$ por $R_{j e} \cdot R_{j n}$.

39 AleXy, A Theory of Constitutional Rights, n. 5 supra, 416.
} 
derecho ambiental, las premisas empíricas cuya verdad está acreditada con toda certeza prácticamente nunca están disponibles. Así, una precedencia absoluta para el principio sustancial de los derechos fundamentales en casos de incertidumbre empírica conduciría a una parálisis legislativa de largo alcance ${ }^{40}$. Lo cual sería una interferencia desproporcionada con el principio formal del legislador democrático. Esta desproporcionalidad se establece por medio de una ponderación de segundo orden, referida a la ponderación de un principio sustancial con un principio formal. En este sentido, se corresponde con el modelo sustancial-formal puro, anteriormente presentado. Pero éste es un caso especial de ponderación sustantiva-formal, que tiene lugar no en la fórmula del peso, sino en un meta-nivel, donde interesa la pregunta sobre qué variables y con qué clases de escalas debe insertarse en la fórmula del peso ${ }^{41}$.

Según la ley de los principios en colisión, de la ponderación resulta una relación condicional de precedencia ${ }^{42}$. Los tres escenarios de la escala epistémica pueden considerarse como tales condiciones de precedencia. Si el valor epistémico de un supuesto empírico es fiable o cierto (f), el principio de los derechos fundamentales para el cual esta clasificación es favorable tiene precedencia sobre el principio formal. Esto implica que el núm. $2^{0}$, esto es, 1 , debe insertarse en la fórmula, lo cual significa que el resultado material del lado del derecho fundamental no está en absoluto influenciado por alguna consideración formal o procedimental, como se requiere para los principios formales. $\mathrm{Si}$, sin embargo, el valor epistémico es solamente «plausible» $(p)$ o incluso «no evidentemente falso» $(e)$, el principio formal tiene precedencia en la ponderación de segundo orden sobre el principio material, el cual, si se toma aisladamente, requiere el valor de «fiabilidad» o «certeza» $(f)$ en casos de interferencia. Sin embargo, esta precedencia no determina, como tal, el peso concreto del principio material en colisión $\left(W_{i, j}\right)$. Este peso concreto es determinado por las ocho variables de la fórmula del peso refinada. La precedencia del principio formal en casos de supuestos empíricos simplemente plausibles o incluso no evidentemente falsos sólo significa que estos supuestos no están excluidos de la ponderación. Éstos se admiten con base en el principio formal. Esta admisión, sin embargo, está relacionada con su poder de reducir el peso de los valores sustanciales de las variables del mismo tipo a las que están atados. Los efectos concretos que tiene la inclusión de variables epistémicas en la fórmula del peso, depende de los valores sustituidos por todas las otras variables. El número de combinaciones es grande. Si se toma la fórmula del peso refinada con cuatro variables de cada lado, es decir, con ocho variables en total, se reúnen, en la base de las dos escalas triádicas, $3^{8}$,

40 Ibid., 417.

41 En el epílogo de la Teoría de los Derechos Fundamentales he formulado la ley de la combinación la cual opera como sigue: «Los principios formales procedimentales pueden anular principios de derechos fundamentales materiales solo en conexión con otro principio material»; ibid., 423. A primera vista, esto puede leerse como reconocimiento del modelo combinado o modelo mixto sustancial-formal. En una publicación más reciente he propuesto que esta verdaderamente infortunada formulación debe entenderse en una forma en la cual la cláusula "en conexión con" se refiere a una relación de segundo orden de un principio formal hacia una relación de primer orden entre principios materiales»; R. ALEXY, «Comments and Responses», en KLATT (ed.), Institutionalized Reason, n. 1 supra, 331. Esto también requiere aclaración, como una relación de ponderación, el segundo orden de relación está referido a una relación entre principios materiales y formales Esta relación de ponderación de segundo orden está relacionada con la ponderación de primer orden, la cual está determinada profundamente por los resultados de encontrar un balance entre principios formales y materiales en un Segundo nivel, pero en el cual el principio formal no participa directamente.

42 Ibid., 53-54. 
6.561 combinaciones. La ponderación de segundo orden resulta en nada distinto a la puerta de entrada para premisas por debajo del nivel de certeza o de fiabilidad plena para ponderar según las líneas de la fórmula del peso. El rol que éstas juegan allí con respecto al resultado final depende de todos los otros valores.

Incorporar variables materiales así como variables epistémicas en la fórmula del peso no es otra cosa que conectar la primera, esto es, la ley material de la ponderación con la segunda, es decir, la ley epistémica de la ponderación. Es de la mayor significación sistemática que la prótasis de las dos leyes de la ponderación es idéntica. Ambas se refieren al peso de la interferencia con el derecho fundamental definida por la combinación de la intensidad de la interferencia con el peso abstracto, o, más exactamente, por el producto de $I_{i}$ y $W_{i}$. Esto demuestra que el principio de los derechos fundamentales afectado representa el punto arquimédico de la ponderación ${ }^{43}$.

Todo lo anterior habla en contra de enfoques como el de SIECKMANn llamado «modelo de concepciones contrapuestas de derecho» ${ }^{44}$ y el «modelo de dos niveles» ${ }^{45}$ de KLATT y SCHMiDT, el cual separa la ponderación de los principios materiales de la ponderación de los principios formales. En el segundo orden o meta-ponderación, la ponderación de los principios formales es, necesariamente, una ponderación con el principio material en colisión ${ }^{46}$, y con el resultado de esta ponderación, la incorporación de variables epistémicas y sus escalas en la fórmula del peso, los principios formales estarían representados en la ponderación de primer orden según esta fórmula.

\section{PRINCIPIOS FORMALES Y DISCRECIONALIDAD}

\section{Martin BOROWSKI ha sostenido que:}

La variable «R», que Alexy introduce en la fórmula del peso, no es un principio formal ni genera discrecionalidad ${ }^{47}$.

Es cierto, de hecho, que la variable $R$ no es un principio formal. Pero es falso que no genera discrecionalidad. La decisión sobre el cannabis de la Corte Constitucional Federal Alemana podría servir para explicar esto. La Corte considera que el supuesto establecido por el legislador sobre el gran peligro asociado a los productos derivados del cannabis es bastante justificable o plausible. De otro lado, concede la tesis contraria, específicamente, que el peligro es mínimo, como sostuvo el juez SOMMER en su voto particular ${ }^{48}$, de manera que ésta sería también plausible. Esto significa que la Corte consideró plausibles las dos premisas, es decir, la del mayor peligro y la del peligro menor $(p)$. Estas clasificaciones epistémicas, ambas, conciernen a la variable $R_{j}$

43 Ibid., 419.

44 J. Sieckmann, Recht als normatives System, Baden-Baden, Nomos, 2009, 200-204.

45 KLATT y SCHMIDT, «Epistemic discretion in constitutional law», n. 16 supra, 100.

46 Es de resaltar que SIECKMANN describe la ponderación en este Segundo escenario como ponderación de principios formales en la cual, sin embargo, los principios materiales pueden jugar un papel: «de otro lado, los principios materiales pueden hacerse relevantes a pesar de los principios formales. Una alta intensidad de interferencia con un derecho fundamental, puede, por ejemplo, justificar un control constitucional más extensivo»; SIECKMANN, Recht als normatives System, n. 44 supra.

47 BorowsKI, «Formelle Prinzipien und Gewichtsformel», n. 3 supra, 174.

48 BverfGE 90, 145 (220). 
en la fórmula del peso. La premisa del mayor peligro y la del peligro menor son la base de clasificaciones materiales bastante diferentes en relación con la importancia de la interferencia con el derecho fundamental afectado, en este caso, con el derecho a la libertad, es decir, éstas conducen a diferentes valores de la variable $I_{j}$. El supuesto del peligro mayor requiere el valor grave $(g)$, el supuesto del menor peligro el valor leve $(l)$. Si se asume: primero, que los valores abstractos, $W_{i}$ y $W_{j}$, son iguales, es decir, que pueden reducirse; segundo, que la intensidad de interferencia con la libertad es moderada $(m)$; tercero, que es fiable o cierto $(f)$ que esta interferencia es causada por la prohibición sobre los productos de cannabis; y cuarto, que los valores normativos epistémicos no juegan ningún papel; se obtienen los siguientes dos resultados al insertar todo esto en la fórmula del peso. En el caso del supuesto del mayor peligro opera así:

$$
W_{i, j}=\frac{2 \cdot 1}{4 \cdot \frac{1}{2}}=1
$$

Con el peso concreto 1, el resultado es un punto muerto, esto es, que el legislador tiene discrecionalidad estructural. La prohibición sobre los productos de cannabis permanece dentro de la discrecionalidad del legislador. Esto es, por tanto, constitucional. El supuesto del peligro menor conduce hacia el resultado opuesto. En números:

$$
W_{i, j}=\frac{2 \cdot 1}{1 \cdot \frac{1}{2}}=4
$$

El núm. 4 expresa que el derecho fundamental tiene precedencia sobre el peligro a combatir. Lo cual significa que la prohibición sobre los productos de cannabis es desproporcional, y por ello, inconstitucional.

Según BorowsKi, la inserción de la variable $R$ en la fórmula del peso no establece discrecionalidad. Nuestro ejemplo, sin embargo, muestra de qué forma al insertar esta variable en la fórmula del peso se establece discrecionalidad. Cuando dos supuesto opuestos tienen el mismo valor epistémico, en esta caso el valor plausible ( $p$ ), esto es, $\frac{1}{2}$, entonces existe un punto muerto epistémico. Ahora lo decisivo es que en los casos de punto muerto epistémico el legislador tiene discrecionalidad epistémica. Con respecto a la decisión del cannabis esto significa que el legislador es libre de decidir si se desea seguir por la línea del peligro mayor, es decir, concebir que la prohibición es constitucional, o seguir la línea del peligro menor y concluir que la prohibición es inconstitucional ${ }^{49}$. Con esto, la variable $R$, de hecho, establece discrecionalidad. Lo

49 KLATT y SCHMIDT han argumentado que esta reconstrucción de la decisión sobre el cannabis concede «al legislador una facultad discrecional de clasificación que es demasiado amplia»; KLATT y SCHMIDT, «Discreción epistémica en el derecho constitucional», n. 16 supra, 102. Esta objeción se basa en su tesis de que la ponderación ya es necesaria en el nivel de clasificación de las intensidades de interferencias. Ellos llaman a esto la «ponderación de clasificación»; ibid., 81. Según KLATT y SCHMIDT, esta ponderación de clasificación trae como resultado que el legislador tiene en el caso del cannabis discrecionalidad no epistémica. El legislador debe insertar en la fórmula del peso el valor grave $(g)$ por la importancia concreta de los peligros relacionados con el fármaco, es decir, que tiene que seguir la tesis del peligro mayor cuando se determina el valor de $I_{j} ;$ ibid., 83. Los dos autores son muy conscientes de que los valores epistémicos para la tesis del mayor peligro, así como para la tesis del peligro menor son los mismos. Sin embargo, excluyen la discrecionalidad sobre la base de diferentes grados de peligro, es decir, sobre el contenido de los dos supuestos contrapuestos. Esto parece, sin embargo, 
hace en todos los casos de punto muerto epistémico. Si el principio formal no existiera, el principio material de los derechos fundamentales excluiría cualquier interferencia sobre la base de premisas simplemente plausibles o no evidentemente falsas, o requeriría que la premisa más favorable se adoptara siempre, es decir, en el caso cannabis, la premisa del peligro menor. Es más, esta discrecionalidad es indirectamente ${ }^{50}$ establecida por el principio del legislador legitimado democráticamente. Este principio formal requiere que la fiabilidad de la variable $R$ se inserte en la fórmula del peso. Mucho más podría decirse acerca del rol de los principios formales en la ponderación. Pero lo que se ha presentado aquí es suficiente para indicar cómo éstos pueden influenciar la ponderación de los derechos fundamentales sin convertirse o en víctima de Escila con la combinación del modelo sustancial-formal, es decir, el modelo que simplemente adiciona principios formales en la fórmula del peso, o en víctima de Caribdis con el modelo de la separación, el cual intenta dar lugar a la ponderación de los principios formales en un escenario en el que los principios materiales no están en casa.

(Traducción del original inglés de María Claudia Quimbayo Duarte)

no ser plausible para hacer la selección de los supuestos con valores epistémicos iguales que dependen de su contenido. Su contenido es un factor que tiene que insertarse en la fórmula del peso, que lo conecta con todos los otros aspectos relevantes. Vid. también M. KLATT y J. ScHMIDT, Spielräume im öffentlichen Recht, Tübingen, Mohr Siebeck, 2010, 25-27.

50 Vid. Alexy, «Comments and Responses», n. 41 supra, 331. 